Henri Labayle

\title{
Judiciarisation, droits fondamentaux et espace pénal européen*
}

\begin{abstract}
Summary
The 'judicialisation' of the European criminal area in perspective with fundamental rights sets various principle as well as functioning issues. The judicial control's upgrade brought about by the Lisbon treaty and the perspective of the EU's accession to the ECHR do not necessarily guarantee a satisfactory answer to the needs of a common criminal area. The EU judge's 'constitutional' function is not enough to make up for the shortcomings of its 'judicial' function, which is confirmed by a statistical assessment of the case law. And yet, the process of mutual recognition between the national criminal systems and the operational role given to agencies call for a judicial protection of individuals which has to be reflected upon despite the considerable obstacles it raises.
\end{abstract}

\section{Zusammenfassung}

Die Verrechtlichung des Europäischen Strafrechtsraums mit Blick auf die Grundrechte bringt sowohl verschiedenen Prinzipien-als auch Funktionsfragen mit sich. Die Aufwertung justizieller Kontrolle, wie sie durch den Lissabonner Vertrag und durch den bevorstehenden Beitritt der EU zur Europäischen Menschenrechtskonvention beding ist, garantiert nicht zwingend eine befriedigende Antwort auf die Bedürfnisse eines gemeinsamen Strafrechtsraums. Die Verfassungsfunktion eines EU-Richters ist nicht hinreichend, um die Unzulänglichkeiten seiner justiziellen Schutzfunktion auszugleichenwas durch eine statistische Analyse von Einzelfällen bestätigt wird. Darüber hinaus verlangen der Prozess der gegenseitigen Anerkennung zwischen den nationalen Kriminaljustizsystemen und die operationelle Rolle, die man EU-Organen zuschreibt, nach richterlichem Schutz von Individuen. Über diesen müssen wir nachdenken, mag er auch auf beträchtliche Hindernisse treffen.

Toute réflexion sur la « judiciarisation » de l'espace pénal européen oblige à plonger dans l'histoire de cet espace. Qu'il s'agisse de sa genèse ou de son avenir immédiat, la place du juge au sein de l'espace répressif européen constitue depuis ses origines un enjeu majeur de cet espace, sans que l'on en perçoive nécessairement toutes les impli-

* Ce texte reprend celui d'une intervention au $\mathrm{VI}^{\circ} \mathrm{VI}^{\circ}$ Forum des Juristes européens, Luxembourg, 2011. 
cations. ${ }^{1}$ 《 Juridictionnalisation » et « judiciarisation » ont ainsi souvent été mises en avant pour souligner l'aspiration à voir l'Union privilégier le règlement judiciaire au traitement diplomatique des litiges, la seconde idée renvoyant plus clairement à un droit de l'individu au règlement judiciaire que la première. Intégrer le juge au sein de l'espace pénal européen n'a pas exactement la même connotation selon que ce juge a pour tâche de réguler l'application du droit commun ou de garantir les droits individuels.

La logique, d'abord, a conduit immédiatement à une conclusion cruelle : comment imaginer une « Europe judiciaire » sans juges... L'imagination des chancelleries fut donc sollicitée, de Schengen à Maastricht, pour persuader les opinions publiques du bien-fondé de ce refus de la « judiciarisation $»^{2}$ de l'Espace de liberté, sécurité et justice (ELSJ). Acté par les traités constitutifs puis démenti avec réticences par le traité d'Amsterdam, ce refus fit gaspiller près de vingt années avant que le juge de l'Union se voie reconnaître à Lisbonne un droit de cité entier au cœur de l'espace de liberté, sécurité et justice. ${ }^{3}$ Ce combat pour un principe a malheureusement dispensé d'une réflexion sur le fond qui conserve toute son actualité, l'essentiel des tentations d'un Etat membre, le Royaume Uni, de se dégager de l'ELSJ se nourrissant de sa prévention à l'encontre du juge de l'Union.

Le poids des habitudes, ensuite, masque encore une partie des enjeux. La lenteur avec laquelle l'espace pénal européen conquis son autonomie autant que la tentation naturelle des débuts à reproduire les schémas classiques de l'entraide répressive internationale où le juge national était le seul interlocuteur judiciaire, ont retardé le moment de réfléchir sérieusement à « la judiciarisation » de l'espace pénal. Les développements originaux de cet espace pénal, de la reconnaissance mutuelle à l'action opérationnelle, ont obligé tardivement les maîtres des traités que sont les Etats membres à reconsidérer les choses. $^{4}$

Cette réflexion est en passe aujourd'hui d'être renouvelée par la montée en puissance de la garantie des droits fondamentaux dans les sociétés nationales comme européennes. L'impact de la Convention européenne des droits de l'Homme se cumule à la justiciabilité d'une Charte européenne des droits de l'Homme et oblige à un constat de carence :

1 Voir notre étude, «Le juge de l'Espace de liberté, sécurité et justice de l'Union européenne », Mélanges en l'honneur du président Genevois, Dalloz, 2009 p. 591. Voir également : S. de Biolley, «Un pouvoir juridictionnel européen en matière pénale? », Actualités de droit pénal européen, Revue de droit pénal et de criminologie, La Charte, 2003, p. 195; D. Flore et S. de Biolley, " Des organes juridictionnels en matière pénale pour l'Union européenne » CDE 2003 p. 596; Le contrôle juridictionnel dans l'espace pénal européen, S. Braum et A. Weyembergh (dir.), ed. de l'ULB, 2009; Cour de justice et justice pénale en Europe, G. GiudicelliDelage et S. Manacorda (dir.) Société de législation comparée, Paris, 2010 et notamment A. Nieto-Martin, "Architectures judiciaires de l'espace pénal européen" p. 271; V. Covolo, "Et la judiciarisation de l'espace pénal fut ... mais où se cache le juge pénal européen?", CDE.

2 Pendant que, très tôt, le pouvoir politique proposait, à l'image de R. Badinter au début des années quatre-vingt, d'explorer la voie d'une juridiction pénale européenne.

3 Voir V. Hatzopoulos, "Casual but smart : the Court's new clothes in the Area of Freedom, Security and Justice after the Lisbon Treaty » in J. Monar (dir.), The institutional dimension of the EU « Area of Freedom, Security and Justice«, PIE Peter Lang, Bruxelles, 2009.

4 Voir sur ce thème G. Bachoué, « Le contrôle juridictionnel de la coopération intergouvernementale dans l'Union, contribution au processus de juridictionnalisation de l'Union ", Thèse, Bayonne, 2012. 
l'intégration européenne comportant l'abolition des frontières intérieures et la construction d'un espace répressif commun ne s'est pas accompagnée d'un renforcement des garanties fondamentales à la hauteur des défis engendrés par cette expérience sans précédent. $^{5}$

La « judiciarisation » de l'espace pénal européen oblige en effet à aller au delà des approches classiques centrées sur la place institutionnelle du juge. Certes, cette place est bien toujours centrale au sein d'une Communauté de droit. Néanmoins, l'impasse sur une réflexion de type fonctionnel, attachée à l'implication quotidienne du juge dans le fonctionnement de cet espace pénal, n'est plus possible. Confiner le débat sur la place du juge dans l'espace pénal européen à une simple fonction de régulation, fut-elle d'envergure constitutionnelle, n'est plus satisfaisant. Parce que les particularités de l'action du juge pénal l'exigent, y compris au plan européen, sans doute est-il bon de comprendre le double mouvement qui caractérise aujourd'hui l'espace pénal européen.

D'abord, la terminaison d'une phase décisive de sa construction marque une forme d'aboutissement : la « judiciarisation » de l'espace répressif européen est désormais admise au sens où le traitement juridictionnel de cet espace est acté, traduit dans les dispositions du traité de Lisbonne (I). Cet acquis est loin de clore le débat, bien au contraire. Il provoque une interrogation majeure : à quelle forme de judiciarisation doit être soumis l'espace pénal européen pour que soient satisfaites les exigences d'une Communauté de droit et garanti un fonctionnement harmonieux (II)?

\section{L'admission du contrôle juridictionnel de l'espace penal europeen}

Les réticences à admettre le juge au sein de l'espace pénal européen n'ont pas été sans conséquences. L'ouverture progressive vers une « judiciarisation » de cet espace s'est réalisée au prix d'une grande complexité (1.) et elle demeure entourée d'une certaine ambiguïté des fonctions confiées à ce juge (2.).

\section{La complexité de la judiciarisation de l'espace pénal européen}

L'un des résultats majeurs de la négociation du traité de Lisbonne tient dans l'alignement du contrôle juridictionnel de l'espace pénal européen sur le droit commun qu'il réalise. Il n'en simplifie pas pour autant l'architecture.

\section{- La mise à niveau opérée par le traité de Lisbonne}

Le traité de Lisbonne, dans sa version TFUE, met fin aux particularités du contrôle juridictionnel portant sur l'Espace de liberté, sécurité et justice (ELSJ). Cela vaut pour sa dimension migratoire à propos de la collaboration préjudicielle et, surtout, de l'espace pénal commun. Deux facteurs concourent à ce résultat. L'un est propre à l'ELSJ, dont le traitement est désormais banalisé. L'autre est plus général puisque l'espace pénal

5 F. Ost, «Quand l'enfer se pave de bonnes intentions... À propos de la relation ambivalente du droit pénal et des droits de l'Homme », in Y. Cartuyvels, H. Dumony, F. Ost, F. Van Drooghenbroeck (dir.), Les droits de l'Homme, bouclier ou épée du droit pénal? Bruylant, Bruxelles, 2007, p. 7. 
européen profite des réponses du nouveau traité aux critiques frappant le déficit de la protection juridictionnelle effective dans l'Union.

- Mettre fin au particularisme contentieux de l'espace pénal européen était nécessaire. Ce choix accompagne la disparition de la construction de l'Union européenne en « piliers » dont l'une des justifications était, justement, de restreindre la place du juge de l'Union européenne. Aussi, le TFUE soumet l'espace pénal européen au jeu du contentieux ordinaire. Disparaît donc l'ancien article 35 TUE qui ménageait une organisation judiciaire particulière, à mi-chemin entre adaptation, déformation et expérimentation. A l'adaptation se rattachaient les aménagements de la collaboration préjudicielle qui n'ont pourtant pas empêché la Cour de justice de céder au « vent du large $\aleph^{6}$ en s'avérant un acteur principal de l'espace pénal européen. A la volonté de déformation des mécanismes contentieux communautaires, devait être imputée l'exclusion du Parlement européen et des citoyens du contentieux de l'annulation du droit dérivé, une volonté identique expliquant le refus de voir le contentieux du manquement trouver sa place dans un univers qui en avait pourtant cruellement besoin. Enfin, la curiosité provoquée par le recours dans l'intérêt de la loi institué par le dernier paragraphe de l'article 35 TUE demeurera éternellement insatisfaite puisque ce dernier n'a jamais trouvé matière à application...

Désormais, le contrôle juridictionnel de l'espace pénal européen obéira aux règles de droit commun du traité, à quelques précisions près. La première est de taille puisque le futur dispositif n'entrera en application que cinq années après l'entrée en vigueur du traité de Lisbonne, c'est à dire le 30 novembre 2014. En vertu de l'article $10 \S 1 \mathrm{du}$ protocole 36 sur les dispositions transitoires, l'article 35 TUE restera en effet applicable jusque là. La seconde précision n'est négligeable non plus puisque l'article 2 du protocole 21 qui organise la position des îles britanniques à l'égard de l'ELSJ indique qu'aucune des décisions de la Cour de justice concernant des actes à propos desquels ces Etats membres auraient exercé leur «opt-out» ne leur est applicable. D'où leur réflexion actuelle sur un éventuel désengagement de l'ensemble du droit adopté depuis 1992.

Pour le reste, et c'est là l'essentiel, le jeu normal du recours en annulation, en indemnité, en carence et, surtout, en manquement ${ }^{7}$ sera ouvert à ses titulaires respectifs, c'està-dire y compris aux particuliers en matière d'annulation. De même, le renvoi préjudiciel retrouvera-t-il les caractéristiques qui lui ont permis d'obtenir le succès que l'on sait en contribuant à l'uniformité d'interprétation et d'application du droit de l'Union. Cette remise à niveau de la collaboration préjudicielle est décisive au regard de la fonction du renvoi préjudiciel, mettre en forme le dialogue des juges, comme à celui de son utilité, obtenir une lecture uniforme du droit positif, délivrée de toute géométrie variable comme à Amsterdam. Que cette dernière n'ait pas véritablement fait l'objet de débats visant à la perpétuer est d'ailleurs significatif. Les Etats membres en situation de dérogation

6 JC Bonichot, « Union européenne et droit pénal : le vent du large? », Mélanges en l’honneur du président Genevois, Dalloz, 2009 p. 75.

7 La question n'est pas d'école pour l'espace pénal européen. La lecture du rapport d'audience de l'affaire Pupino où les Etats membres mettaient ouvertement en doute l'autorité et la portée des décisions-cadre de l'article $34 \mathrm{du}$ TUE illustre à quel point l'intervention du juge au titre de la constatation de manquement est indispensable pour garantir le fonctionnement de l'ELSJ. 
comme les nouveaux Etats membres se trouveront donc désormais sur un pied d'égalité au sein de l'Union.

- Ce retour à l'ordinaire du contentieux européen comporte des conséquences importantes pour le droit de l'espace pénal européen et, plus généralement, pour les mécanismes de coopération intergouvernementale.

En premier lieu et au plan matériel, l'immunité contentieuse du pilier PESC expliquait que les constructions sécuritaires de l'Union en matière de lutte contre le terrorisme y aient trouvé abri, la Cour ayant manifesté à plusieurs reprises sa défiance à cet égard. Cette sanctuarisation est désormais entamée par l'article 275 TFUE. Ce dernier dispose que « la Cour de justice de l'Union européenne n'est pas compétente en ce qui concerne les dispositions relatives à la politique étrangère et de sécurité commune, ni en ce qui concerne les actes adoptés sur leur base. Toutefois, la Cour est compétente pour contrôler le respect de l'article 40 du traité sur l'Union européenne et se prononcer sur les recours, formés dans les conditions prévues à l'article 263, quatrième alinéa, du présent traité concernant le contrôle de la légalité des décisions prévoyant des mesures restrictives à l'encontre de personnes physiques ou morales adoptées par le Conseil sur la base du titre V, chapitre 2, du traité sur l'Union européenne ». Autrement dit, le respect de l'intégrité des compétences de l'Union comme la protection des individus seront garantis par le juge de l'Union même en cette matière si particulière. Preuve de l'attention portée à la question, une Déclaration jointe au traité insiste sur la garantie d'un «contrôle juridictionnel rigoureux $\gg{ }^{8}$

Ensuite, au plan technique, l'espace pénal européen bénéficie de l'amélioration générale du contrôle de la légalité du droit dérivé de l'Union, du fait de la réforme de l'accès des particuliers au recours en annulation du nouvel article 263 TFUE. La Cour de justice s'était refusée à un élargissement sur ce point par voie jurisprudentielle dans les contentieux UPA et Jego-Quéré en raison du verrou textuel de l'intérêt « direct et individuel » exigé par le traité. Beaucoup était attendu d'un nouveau traité et deux tendances s'opposaient. D'une part, les tenants du statu quo faisaient valoir que les standards « essentiels » d'une protection juridictionnelle effective étaient respectés par l'Union européenne dans la mesure où la subsidiarité juridictionnelle confie aux juridictions nationales le soin de garantir les droits des particuliers. D'autre part, la volonté d'ajuster à la marge les conditions de recevabilité des recours des particuliers avait également ses partisans. De l'ambition d'un recours spécifique en matière de protection des droits fondamentaux, à la mention des effets de l'acte contesté sur la situation juridique du requérant ou à la prise en considération de l'existence ou non de mesures d'exécution

8 Déclaration 25 relative aux articles 75 et 215 du TFUE : «La Conférence rappelle que le respect des droits et des libertés fondamentaux implique notamment qu'une attention suffisante soit accordée à la protection et au respect du droit des personnes physiques ou des entités concernées de bénéficier des garanties prévues par la loi. À cette fin, et afin de garantir un contrôle juridictionnel rigoureux des décisions soumettant une personne physique ou une entité à des mesures restrictives, les décisions en question doivent s'appuyer sur ces critères clairs et distincts. Ces critères devraient être adaptés aux caractéristiques propres à chaque mesure restrictive». 
de l'acte contesté, plusieurs voies avaient été explorées lors des travaux de la Convention. ${ }^{9}$ Les auteurs du traité se sont rangés à la seconde approche, celle de l'ajustement.

L'article 263 TFUE réserve le jeu du recours en annulation à des requérants " privilégiés » ou « constitutionnels » dont les particuliers ne font pas partie. Néanmoins son paragraphe 4 procède à une modification en affirmant que «toute personne physique ou morale peut former, dans les conditions prévues au paragraphes 1 et 2 , un recours contre les actes dont elle est le destinataire ou qui la concernent directement et individuellement, ainsi que contre les actes réglementaires qui la concernent directement et qui ne comportent pas de mesures d'exécution ».

Enfin, un apport important s'y ajoute. L'article $263 \S 1$ TFUE précise que la Cour « contrôle aussi la légalité des actes des organes ou organismes de l'Union destinés à produire des effets juridiques à l'égard des tiers », même si le paragraphe 5 de ce même article précise que les actes instituant ces acteurs "peuvent prévoir des conditions et modalités particulières concernant les recours formés par des personnes physiques ou morales contre des actes de ces organes ou organismes destinés à produire des effets juridiques à leur égard ». Les critiques adressées à des acteurs majeurs de l'entraide répressive tels qu'Europol devraient donc s'atténuer ${ }^{10}$ et la question du contrôle juridictionnel des actes d'Eurojust ou du futur Procureur européen est posée ouvertement.

- La singularité du contrôle juridictionnel de l'espace pénal européen conserve cependant des marques fortes. Comme le traité d'Amsterdam, l'article 276 du TFUE spécifie à nouveau que « dans l'exercice de ses attributions concernant les dispositions des chapitres $I V$ et $V$ du titre $V$ relatives à l'espace de liberté, de sécurité et de justice, la Cour de justice de l'Union européenne n'est pas compétente pour vérifier la validité ou la proportionnalité d'opérations menées par la police ou d'autres services répressifs dans un État membre, ni pour statuer sur l'exercice des responsabilités qui incombent aux États membres pour le maintien de l'ordre public et la sauvegarde de la sécurité intérieure ».

\section{- L'architecture du contrôle juridictionnel de l'espace pénal}

L'architecture du contrôle juridictionnel de l'Union est dépourvue de simplicité de manière générale et cette situation ne s'améliore pas lorsque la matière pénale entre en jeu. La « juridiction » de l'Union présente des caractéristiques à nulle autre pareille.

- A cet égard, l'originalité de la relation établie par la voie préjudicielle entre le juge de l'Union et le juge national s'avère déterminante pour la diffusion et l'unité d'application du droit commun, chacun le sait.

La Cour y est extrêmement attachée et elle vient encore de le souligner avec force. L'avis 1/09 rendu dans l'affaire dite de la Juridiction du brevet européen ${ }^{11}$ illustre bien la conception qu'elle s'en fait et son refus d'y déroger. La Cour de justice y saisit l'occasion de souligner qu'il « incombe aux juridictions nationales et à la Cour de garantir la plein application du droit de l'Union dans l'ensemble des Etats membres ainsi que

9 Dont celle consistant à remplacer le « et » de la formule « directement et individuellement " par un « ou » qui n'exigerait plus le cumul.

10 Voir également CJCE, 15 mars 2005, Espagne/Eurojust, C-160/03, Rec. II-981.

11 CJUE, 8 mars 2011, avis 1/09. 
la protection juridictionnelle des droits que les justiciables tirent dudit droit... en effet le juge national remplit en collaboration avec la Cour une fonction qui leur est attribuée en commun en vue d'assurer le respect du droit dans l'interprétation et l'application des traités... ». ${ }^{12}$ Cela la conduit à qualifier fortement la collaboration préjudicielle et à refuser qu'il y soit porté atteinte sous peine de voir " dénaturer » les compétences fixées par le traité. En effet, «le système instauré à l'article 267 TFUE établit une coopération directe entre la Cour et les juridictions nationales ... et les fonctions attribuées respectivement aux juridictions nationales et à la Cour sont essentielles à la nature même du droit institué par les traités ». ${ }^{13}$

- Cependant, la crise fait, ici aussi, son office et le système juridictionnel de l'Union peine à la surmonter, de Nice à Lisbonne. Crise de la relation préjudicielle, tout d'abord, en raison de la lenteur avec laquelle la vingtaine de mois nécessaires à une réponse affecte la tenue de l'instance nationale et au vu des réticences de la Cour à céder une part de son exclusivité en la matière. Crise de fonctionnement ensuite, liée à un succès qui ne se dément pas et dont les progrès de productivité de la juridiction peinent à compenser les effets. ${ }^{14}$ L'espace pénal a toutes chances d'en subir les inconvénients, sauf, ce qui demande à être examiné, si le renvoi préjudiciel ne joue pas dans l'espace pénal européen le rôle qu'il tient dans les autres domaines de l'intégration européenne, et l'on y viendra plus loin.

Le traité de Lisbonne n'apporte pas de réponse véritablement convaincante à cet égard. Certes, la création de «tribunaux spécialisés » établis par voie de règlement et adjoints au Tribunal de première instance est une nouveauté. Le Traité de Nice avait néanmoins déjà introduit une base juridique nécessaire à la création de « chambres juridictionnelles », chargées de connaître en première instance de certaines catégories de recours formés dans des matières spécifiques. Sans succès. Pour le reste, la stabilité semble de mise, y compris et notamment à propos de la fonction préjudicielle que certains avaient espéré voir décentralisée.

- Pour ce qui est de l'espace pénal européen, le juge national y occupe une place très particulière. Situé dans des rapports de nature horizontale avec les autres juges nationaux qu'il sollicitait ou qui le sollicitaient, le juge interne y animait l'entraide répressive classique et notamment le droit de l'extradition. L'absence de relation hiérarchique permettait ainsi la coopération à condition que la confiance respective existe entre les partenaires.

L'espace pénal de l'Union européenne renouvelle cette lecture, dépassant la simple existence de rapports horizontaux. Le juge national y est et demeure un acteur à part entière du fonctionnement de cet espace, à des degrés divers qui appellent vraisemblablement autant de réponses particulières. Il n'est pas certain en soi que les besoins très particuliers de l'entraide répressive judiciaire trouvent dans l'organisation contentieuse actuelle les réponses attendues. De plus, un certain nombre de choix opérés à Lisbonne laissent d'ores et déjà penser que l'horizontalité de ces relations est battue en brèche : fonder la coopération judiciaire pénale sur la reconnaissance mutuelle appellera une

12 points 68 et 69 .

13 points 84 et 85 .

14 Le président Skouris note ainsi dans la présentation du Rapport général d'activités 2010 qu'un total de 1406 affaires ont été introduites auprès des trois juridictions qui composent la Cour de justice, ce qui représente le chiffre le plus élevé dans l'histoire de l'institution. 
régulation, l'appuyer sur des mécanismes opérationnels tels que le Réseau judiciaire européen ou Eurojust et, demain peut être, sur un Procureur européen, renforcent les interrogations. Elles poussent à douter de la capacité de l'architecture actuelle de l'espace pénal européen à répondre aux défis posés.

\section{L’ambiguïté de la judiciarisation de l'espace pénal européen}

Le descriptif du contrôle juridictionnel de l'espace pénal européen est connu. Son ambiguïté structurelle l'est sans doute moins : faisant la place belle à une fonction régulatrice de nature « constitutionnelle », cette orientation occulte les besoins nouveaux de la « judiciarisation » de l'espace pénal européen. ${ }^{15}$

\section{- La prépondérance de la fonction constitutionnelle}

La pluralité des rôles confiés à la Cour de justice par les fondateurs de l'Union, juge de droit commun du droit de l'Union mais aussi juge international ou constitutionnel, offre une gamme de possibilités sans équivalent. Pour ce qui est de la gestion de l'espace pénal européen, apparu récemment, la fonction constitutionnelle l'emporte encore jusqu'ici sur les autres.

- L'état des lieux imposait cette priorité donnée à la démarche constitutionnelle. Une intervention de cette nature demeure encore indispensable, à des fins de régulation institutionnelle d'un dispositif suscitant structurellement des conflits institutionnels.

Le contentieux de la base juridique est significatif de ce besoin fondamental éprouvé par l'espace pénal européen. Entamé avec la querelle sur la communautarisation du droit pénal européen ${ }^{16}$ et poursuivi avec le cadrage de la lutte contre le terrorisme, ${ }^{17}$ le travail de délimitation opéré par la Cour s'est avéré décisif sur le plan technique, certes, mais aussi et surtout sur le plan des droits fondamentaux. L'un des acquis les plus remarquables de la jurisprudence $P N R$ ne fut-il pas de provoquer l'adoption d'un texte garantissant la protection des données dans le cadre de l'ex-troisième pilier qui en était précisément dépourvu ${ }^{18}$ ? En d'autres termes, l'intervention de l'Union sur le terrain de la lutte contre le terrorisme s'est traduite par une progression des droits fondamentaux au même titre d'ailleurs que le contentieux des listes anti-terroristes de l'Union...

Cette intervention ne relève pas de la seule histoire de l'espace pénal européen et le traité de Lisbonne appellera encore des arbitrages de ce type. La connexion de l'espace pénal européen avec la politique étrangère et de sécurité commune constitue l'un des enjeux les plus visibles ${ }^{19}$ de ces débats tout comme la question de la participation un-

15 Sur le juge, voir F. Ost, « Jupiter, Hercule, Hermès : trois modèles du juge », in F. Ost, Dire le droit, faire justice, Bruylant, Bruxelles, 2007, pp. 32-60.

16 H. Labayle, «L'ouverture de la jarre de Pandore, réflexions sur la compétence de la Communauté en matière pénale », CDE 2006 p. 379.

17 H. Labayle et R. Mehdi, « Le contrôle juridictionnel de la lutte contre le terrorisme : les «blacks lists« de l'Union dans le prétoire de la Cour de justice » RTDE 2009 p. 231.

18 Décision cadre 2008/977 du 27 novembre 2008 relative à la protection des données à caractère personnel traitées dans le cadre de la coopération policière et judiciaire en matière pénale, JO L 350 du 30 décembre 2008 p. 60.

19 Voir par exemple en ce qui concerne le rattachement de la lutte contre le terrorisme à la PESC, CJUE, 9 juillet 2012, Parlement c. Conseil, C-130/10. 
anime ou pas des Etats membres à ses développements demeure une question sensible entre toutes. Le positionnement des Etats en situation d'opt-out à l'égard de l'ELSJ aura des conséquences importantes selon que la base juridique de ces développements se situe au sein du Titre V du TFUE ou pas. On sait ainsi que la législation en matière de protection des données, à laquelle l'article 16 TFUE procure une base juridique, est un exemple caractéristique des difficultés à venir, l'opt-out britannique jouant à ce propos en matière de coopération judiciaire pénale et policière...

- Au delà, nombre de régulations institutionnelles demeurent encore à effectuer. A cet égard, l'une des plus importantes concerne vraisemblablement celle de l'article 82 TFUE qui traite de l'intensité normative de la coopération judiciaire pénale en bornant soigneusement celle-ci. Cette dernière n'est envisageable que «dans la mesure où cela est nécessaire pour faciliter la reconnaissance mutuelle des jugements et décisions judiciaires, ainsi que la coopération policière et judiciaire dans les matières pénales ayant une dimension transfrontière » et ne porte que sur des « règles minimales » qui, en tout état de cause « tiennent compte des différences entre les traditions et systèmes juridiques des États membres $» .{ }^{20} \mathrm{~A}$ n'en pas douter, ce dernier terme de phrase est susceptible de réclamer l'interprétation du juge de l'Union, tout comme le fameux mécanisme de « frein/accélérateur » qui s'appuie sur la même logique.

Enfin, la réserve de compétence au profit des Etats membres et concernant les questions de sécurité nationale et d'ordre public laisse présager des querelles tout aussi complexes.

\section{- L'insuffisance de la fonction « judiciaire »}

Privilégier la fonction d'interprétation « constitutionnelle » du juge est naturel, du point de vue de la logique juridique et politique. Ce choix a cependant pour effet implicite de ne pas mettre en avant un besoin spécifique de l'espace pénal européen, celui d'une « judiciarisation » au sens plein du terme. Sans pour autant devenir une "société contentieuse » au sens où les détracteurs d'un « gouvernement des juges » ou les partisans de l'absolutisme du législateur l'entendent, l'espace pénal européen réclame une intervention « judiciaire » plus poussée qu'elle ne l'est actuellement. Des facteurs classiques autant que spécifiques l'expliquent.

- Les conséquences de la « juridicisation » qui caractérise l'espace pénal européen depuis l'admission d'instruments législatifs obligatoires à Amsterdam n'ont sans doute pas été évaluées à leur juste valeur. Elles justifient désormais de marquer plus fortement la présence de l'institution judiciaire. Sans aller jusqu'à une inflation normative, bien loin de là, l'édiction de normes unilatérales européennes en matière pénale réclame une intervention judiciaire correspondant à cette montée en force et répondant aux besoins éprouvés par les particuliers. Ne serait-ce que pour sécuriser les procédures et permettre une stabilité juridique indispensable, cette montée en puissance du juge est souhaitable. Le jeu de la primauté du droit de l'Union autant que son invocabilité par les justiciables, faute de l'applicabilité directe du droit dérivé, justifient cet appel naturel au juge. La particularité de la matière pénale transcende la question, du fait de la prise en considération des droits fondamentaux. Ce mouvement irrépressible explique à la fois que la Cour ait voulu assurer les impératifs d'une protection juridictionnelle effective et que

20 Article 82 § 2 TFUE. 
le jeu de l'entraide répressive soit ordonné ouvertement autour du respect de ces droits fondamentaux.

Il n'est pas indifférent de noter que c'est précisément en matière pénale, le contentieux des listes anti-terroristes, ${ }^{21}$ que la Cour de justice a proclamé de manière éclatante que «le contrôle, par la Cour, de la validité de tout acte communautaire au regard des droits fondamentaux doit être considéré comme l'expression, dans une communauté de droit, d'une garantie constitutionnelle découlant du traité CE en tant que système juridique autonome à laquelle un accord international ne saurait porter atteinte $\gg .{ }^{22}$ Donc, le droit à une protection juridictionnelle effective « constitue une garantie constitutionnelle relevant des fondements mêmes de la Communauté $» .^{23} \mathrm{La}$ Cour a beau jeu à ce propos d'en saluer les sources d'inspiration : « selon une jurisprudence constante, le principe de protection juridictionnelle effective constitue un principe général du droit communautaire, qui découle des traditions constitutionnelles communes aux États membres et qui a été consacré par les articles 6 et 13 de la CEDH, ce principe ayant d'ailleurs été réaffirmé à l'article 47 de la Charte des droits fondamentaux de l'Union européenne, proclamée le 7 décembre 2000 à Nice $\gg .{ }^{24}$ Dès lors, le juge européen permet à l'espace pénal européen de disposer des bases communes indispensables à son fonctionnement, chacun des acteurs ayant la certitude que ce ciment commun consolide l'ensemble.

- Est-ce suffisant pour autant? Sans doute pas, si une véritable ambition anime cet espace commun et ce, pour au moins deux raisons différentes.

La première raison est classique dans le droit de l'entraide répressive internationale. Elle concerne l'articulation des compétences en présence et la solution nécessaire des conflits de juridictions. ${ }^{25}$ Le caractère obligatoire (sinon automatique) des mécanismes d'entraide institués au sein de l'espace pénal européen oblige à garantir la capacité de chaque droit et juge national à accepter sans réticences les sollicitations et décisions du juge étranger. Les contraintes de son propre ordre public et la vision qu'il se fait de la protection des droits fondamentaux peuvent en effet entraver sérieusement l'efficacité du droit commun sans un arbitrage qui ne peut être que judiciaire.

Jusqu'alors, un simple souci d'efficacité dominait la question. Solutionner les différends entre deux systèmes pénaux nationaux à la poursuite d'un coupable permettait de s'assurer que le coupable n'était pas jugé deux fois pour les mêmes faits pas davantage qu'il ne pouvait demeurer impuni. La production jurisprudentielle de la CJUE à ce propos est à la fois ancienne et fournie. Cela s'explique naturellement au vu de la consti-

21 CJUE, 3 septembre 2008, Yassin Abdullah Kadi et Al Barakaat International Foundation c. Conseil et Commission, C-402/05 P et C-415/05 P, Rec. 2008 p. I-6351.

22 point 316.

23 point 290.

24 point 335.

25 Voir la décision-cadre 2009/948 du 30 novembre 2009 relative à la prévention et au règlement des conflits en matière d'exercice de la compétence dans le cadre des procédures pénales, JO L 328 du 15 décembre 2009 p. 42. 
tution d'un espace pénal sans frontières intérieures où le défaut comme la concurrence des compétences respectives font quasiment partie du paysage juridique. ${ }^{26}$

La perspective se fait autre lorsque l'on dépasse la fonction technique du principe ne bis in idem pour prendre en considération le droit fondamental des individus à ne pas être jugé ou condamné deux fois pour les mêmes faits. Garanti par des textes constitutionnels, comme en Italie, internationaux et européens, de la Convention européenne des droits de l'Homme à la Charte des droits fondamentaux, l'exercice de ce droit est susceptible de perturber sérieusement le fonctionnement de l'espace pénal européen s'il n'est pas perçu de façon concordante. Protégeant l'individu contre une double ou même une action répressive multiple, le principe ne bis in idem oblige à réfléchir à " l'unité répressive $»^{27}$ dans cet espace devenu commun car son fondement ultime est la protection de l'individu poursuivi. ${ }^{28}$

Tel était le sens de la brillante démonstration de l'avocat général Bot dans ses conclusions sous l'affaire Mantello, faisant le choix de mettre en avant le caractère de « droit fondamental» d'un principe qui «fait partie de l'ordre juridique de tous les Etats membres, en tant que principe inhérent à la notion d'Etat de droit, et qui a été consacré expressément dans la charte $»{ }^{29}$ D'où un positionnement particulièrement clair du principe : « en prévoyant que le principe ne bis in idem constitue un motif de nonexécution obligatoire et non pas seulement un obstacle à un procès dans l'État membre d'émission, le législateur de l'Union a pris en considération et voulu prévenir les effets attentatoires aux libertés individuelles que produirait l'exécution d'un mandat d'arrêt européen en méconnaissance de ce principe ». Aussi, "l'hypothèse dans laquelle les faits visés par le mandat d'arrêt européen auraient été jugés dans l'État membre d'émission ne constitue pas une situation purement interne, dont la conformité avec les droits fondamentaux devrait être appréciée uniquement à l'aune du droit de cet État membre, sous le contrôle de la Cour européenne des droits de l'homme $» .{ }^{30} \mathrm{On}$ regrettera que la Cour ait fait le choix d'ignorer la portée d'un tel droit fondamental et d'en demeurer, à ce stade, à une lecture notariale de la convention d'application des accords de Schengen et de la décision cadre 2002/584 sur le mandat d'arrêt européen. ${ }^{31}$ Est-on certain que cette lecture aurait été celle de la CEDH?

26 Décision-cadre 2009/948/JAI du 30 novembre 2009 relative à la prévention et au règlement des conflits en matière d'exercice de la compétence dans le cadre des procédures pénales, JO L 328 du 15 décembre 2009 p. 42.

27 J. Lelieur-Fischer, La règle ne bis idem. Du principe de l'autorité de la chose jugée au principe d'unicité de l'action répressive, Thèse, Paris II, 2005.

28 Voir en ce sens G. Taupiac-Nouvel, Le principe de reconnaissance mutuelle des décisions répressives dans l'Union européenne, Toulouse, 2010 p. 297.

29 Conclusions sous CJUE, 16 novembre 2010, Gaetano Mantello, C-261/09.

30 points 86 à 91 .

31 En l'espèce, la juridiction de renvoi demandait si l'article 3.2 de la décisioncadre devait être interprété en ce sens que la circonstance que les enquêteurs, au moment où une personne a été poursuivie et condamnée pour un fait isolé de détention de stupéfiants, disposaient déjà d'éléments de preuve de la participation de cette personne, pendant plusieurs mois, à une association criminelle destinée au trafic de stupéfiants, mais qu'ils n'avaient pas révélés à la juridiction compétente dans l'intérêt de l'enquête, justifiait de considérer que cette participation à l'association criminelle et la détention isolée de stupéfiants constituent les « mêmes faits ». 
La seconde justification d'une évolution nécessaire de l'espace pénal européen vers une " judiciarisation » plus marquée lui est propre. "Fondée » sur la reconnaissance mutuelle selon les termes de l'article 82 TFUE, la coopération judiciaire en matière pénale au sein de l'Union suscite des questions spécifiques du point de vue des droits fondamentaux. En effet, la confiance mutuelle des systèmes pénaux qui composent cet espace pénal et qui commande son fonctionnement ne se décrète pas. Elle commande pourtant le jeu de la reconnaissance mutuelle qui est censée structurer l'espace pénal commun et le jeu d'une confiance « aveugle » ou " détournée » de son objet ne sont, en définitive, pas plus acceptables l'un que l'autre. ${ }^{32}$ D' où le besoin d'un contrôle accru, de nature judiciaire, en même temps que le renforcement des droits procéduraux des suspects ou des personnes poursuivies dans le cadre de procédures pénales. ${ }^{33}$

Le respect des droits fondamentaux applicables en matière pénale constitue la principale source de divergence et donc de défiance mutuelle entre les juges nationaux, dans un univers voué, rappelons-le encore, à la " coopération » et non à l'intégration. La pétition de principe de l'article 6 du traité selon laquelle les Etats membres de l'Union sont respectueux des droits fondamentaux ne suffit pas à surmonter les réticences, bien naturelles au demeurant depuis que la Cour européenne des droits de l'Homme a invité les Etats membres à la vigilance, comme on le verra plus loin.

C'est un fait : les instruments du droit dérivé de l'Union ne permettent pas de faire de l'obligation de respect des droits fondamentaux une condition matérielle de refus de la reconnaissance mutuelle. Soit ils se bornent à souligner dans leur préambule ${ }^{34}$ ou dans le corps du texte que « la présente décision-cadre ne saurait avoir pour effet de modifier l'obligation de respecter les droits fondamentaux et les principes juridiques fondamentaux tels qu'ils sont consacrés par l'article 6 du traité sur l'Union européenne », ${ }^{35}$ soit,

32 Voir A. Weyembergh, «La justice : bilan et perspectives en matière pénale », in C. Kaddous et M. Dony (dir.), D'Amsterdam à Lisbonne, dix ans d'espace de liberté, sécurité et de justice, Buylant, 2010 p. 113.

$33 \mathrm{La}$ « feuille de route » du Conseil établie en la matière est loin de compenser l'échec d'une première tentative en ce sens.

34 Voir le considérant 6 de la décision-cadre 2003/577/JAI du 22 juillet 2003 relative à l'exécution dans l'Union européenne des décisions de gel de biens ou d'éléments de preuves, JOUE L 196 du 2 aout 2003 p. 45 ; le considérant 27 de la décision-cadre 2008/978/JAI du 18 décembre 2008 relative au mandat européen d'obtention de preuves visant à recueillir des objets, des documents et des données en vue de leur utilisation dans le cadre de procédures pénales, JOUE L 350 du 30 décembre 2008 p. 72; le considérant 13 de la décision-cadre 2008/909/JAI du 27 novembre 2008 concernant l'application du principe de reconnaissance mutuelle aux jugements en matière pénale prononçant des peines ou mesures privatives de liberté aux fins de leur exécution dans l'Union européenne, JOUE L 327 du 5 décembre 2008, p. 27.

35 Voir l'article $1 \S 3$ de la décision-cadre 2002/584/JAI du 13 juin 2002 relative au mandat d'arrêt européen et aux procédures de remise entre États membres, JOCE L 190 du18 juillet 2002 p. 1; l'article 1 de la décision-cadre 2008/978/JAI du 18 décembre 2008 relative au mandat européen d'obtention de preuves visant à recueillir des objets, des documents et des données en vue de leur utilisation dans le cadre de procédures pénales, JOUE, L 350 du 30 décembre 2008 p. 72. 
ici où là, des dispositions spécifiques y font allusion. ${ }^{36} \mathrm{C}^{\prime}$ est le cas à propos des règles du procès équitable en matière de mandat d'arrêt européen ${ }^{37}$ comme ailleurs. Le seul texte qui ouvre expressément une possibilité en ce domaine est la décision-cadre relative à l'exécution des sanctions pécuniaires dont l'article $16 \S 3$ affirme que l'Etat d'exécution peut «s'opposer » à la reconnaissance et à l'exécution de la décision «lorsque le certificat visé à l'article 4 donne à penser que des droits fondamentaux ou des principes juridiques fondamentaux définis par l'article 6 du traité ont pu être violés ».

Le respect des droits fondamentaux ne peut donc constituer de manière générale « un motif explicite de refus en cas de violation $»,{ }^{38}$ ce que nombre de systèmes pénaux nationaux éprouvent encore du mal à assimiler et que la Commission n'accepte que de manière " exceptionnelle ». ${ }^{39} \mathrm{Si}$ l'on comprend les deux logiques, celle de l'Etat d'exécution peu soucieux de voir sa responsabilité engagée au titre du respect des droits fondamentaux en s'acquittant de ses obligations, et celle de l'Union européenne désireuse de donner leur effet utile aux textes organisant la reconnaissance mutuelle, les possibilités de blocage n'en restent pas moins certaines.

Le paradoxe vient justement de ce que la reconnaissance mutuelle dans l'Union européenne est désormais caractérisée par une «judiciarisation » rompant avec le passé. La dépolitisation de l'entraide répressive dans l'Union européenne a suffisamment été célébrée lors de l'adoption du mandat d'arrêt européen ${ }^{40}$ pour que l'on souligne le décalage qu'il y aurait à voir se développer une défiance réciproque entre les systèmes répressifs à l'instant où les juges nationaux deviennent des acteurs exclusifs. Aussi, l'espace pénal européen demeure encore à la recherche de son juge. En effet, si le contrôle éventuel du juge national d'exécution est exclu pour des raisons de principe autant

36 Par exemple en ce qui concerne le principe ne bis in idem dont le respect peut constituer un motif de non-reconnaissance ou de non-exécution, voir l'article $7 \S 1$, c) de la décision-cadre 2003/577/JAI du Conseil du 22 juillet 2003 relative à l'exécution dans l'Union européenne des décisions de gel de biens ou d'éléments de preuve, JOUE L 196 du 2 août 2003, p. 45; Voir également les considérants 5 et 6 et les articles 3 et $20 \S 3$ de la décision-cadre 2005/214/ JAI du Conseil du 24 février 2005 concernant l'application du principe de reconnaissance mutuelle aux sanctions pécuniaires, JOUE L 76 du 22 mars 2005, p. 16; les considérants 13 et 14 et l'article $1 \S 2$ de la décision-cadre 2006/783/JAI du Conseil du 6 octobre 2006 relative à l'application du principe de reconnaissance mutuelle aux décisions de confiscation, JO L 328 du 24 novembre 2006; (instrument modifié à présent par la décision-cadre 2009/299/JAI, J.O. L 81 du 27 mars 2009); les considérants 5 et 6 de la décision-cadre 2008/947/JAI du Conseil du 27 novembre 2008 concernant l'application du principe de reconnaissance mutuelle aux jugements et aux décisions de probation aux fins de la surveillance des mesures de probation et des peines de substitution, JOUE L 337 du 16 décembre 2008, p. 102; les considérants 13 et 14 et l'article $3 \S 4$ de la décision-cadre 2008/909/JAI du Conseil du 27 novembre 2008 concernant l'application du principe de reconnaissance mutuelle aux jugements en matière pénale prononçant des peines ou des mesures privatives de liberté aux fins de leur exécution dans l’Union européenne, JOUE L 327 du 5 décembre 2008, p. 27, 38.

37 Voir l'article 11 de la décision-cadre précitée.

38 Voir les rapports d'évaluation de la Commission fondé sur l'article 34 de la décision-cadre du Conseil du 13 juin 2002 relative au mandat d'arrêt européen et aux procédures de remise entre Etats membres, COM (2006) 8, 24 janvier 2006 et COM (2011) 175 du 11 avril 2011.

39 id. \$2.2.3.

40 Voir M. Masse, « La décision-cadre » in ME. Cartier (dir.), Le mandat d'arrêt européen, Bruylant 2005 p. 47. 
que d'efficacité, il ne reste plus alors que le juge européen, celui de l'Union et celui de la CEDH.

La Cour de justice s'aventure avec prudence sur ce terrain délicat en matière pénale, ${ }^{41}$ hors dimension institutionnelle et à la demande du juge national d'exécution. On le conçoit aisément, au vu des accusations d'impérialisme juridique dont elle est fréquemment l'objet. On peut avancer, peut être, aussi que la perspective ne l'enchante guère, même si elle ne s'est pas dérobé à son office.

Le principe de légalité en matière criminelle a été l'une des questions majeures qu'il lui a été donné de connaître dans la mesure où, en fait, sa réponse commandait une bonne part de l'avenir de la reconnaissance mutuelle. L'arrêt Advocaten voor de Wereld rendu le 3 mai 2007 restera l'un des épisodes les plus marquants de cette intervention, en réponse à une question frontale sur le terrain des droits fondamentaux, puisque le juge constitutionnel belge interrogeait la Cour sur la compatibilité de l'énumération effectuée par la décision-cadre avec les exigences du principe de légalité criminelle. Ce dernier implique que soient prédéterminés les comportements illicites et les peines qui y sont attachés. Dans ses conclusions, l'avocat général Jarabo-Colomer avait justement souligné que « cette garantie est le reflet de la sécurité juridique dans le domaine pénal et revêt une importance plus grande en ce qu'elle affecte des valeurs fondamentales telles que la liberté personnelle $\gg .{ }^{42}$

En se plaçant ouvertement sur le terrain des droits fondamentaux et à l'appui de la jurisprudence de la $\mathrm{CEDH}$, la Cour de justice va rappeler que «le principe de légalité des délits et des peines (nullum crimen, nulla poena sine lege), qui fait partie des principes généraux du droit se trouvant à la base des traditions constitutionnelles communes aux États membres, a également été consacré par différents traités internationaux, et notamment à l'article 7 , paragraphe 1 , de la convention européenne de sauvegarde des droits de l'homme et des libertés fondamentales $\gg{ }^{43}$ Dès lors, la validité de la décisioncadre sur laquelle on l'interrogeait est établie. N'instaurant aucune peine ni harmonisation en matière pénale, l'instrument qu'est le mandat d'arrêt européen se borne à structurer un mécanisme d'entraide entre juges de différents pays dans une procédure lorsqu'il s'agit de déterminer qui a commis un délit ou lorsqu'il s'agit d'assurer l'exécution d'une condamnation, le tout sous certaines conditions.

De façon moins directe, puisque la Cour était sollicitée par voie d'interprétation, elle manifeste le même souci dans toute une série d'affaires, n'hésitant pas à se dégager d'une interprétation stricte au bénéfice des droits fondamentaux de la personne intéressée comme dans l'affaire $I B .{ }^{44}$

Il reste que, comme on va y revenir, la rareté des interrogations qui parviennent à la Cour et la pauvreté des questions qui lui sont soumises laissent dubitatif sur la suite. Il convient d'aller au delà en poussant la réflexion sur la nature de cette judiciarisation.

41 A. Weyembergh, « Le principe ne bis in idem : pierre d'achoppement de l'espace pénal européen » CDE 2004 p. 337 et la chronique de jurisprudence relative à l'ELSJ tenue avec S. de Biolley, CDE 2010 p. 166.

42 point 102.

43 point 49.

44 CJUE, 21 octobre 2010, I.B. C-306/09, commentaire A. Weyembergh, JDE 2011 p. 71. 


\section{L'exercice du controle juridictionnel de l'espace penal europeen}

Si l'on admet la nécessité d'accroître la pression judiciaire sur l'espace pénal européen, deux questions s'imposent à l'analyse : celle de l'articulation du contrôle effectué au plan interne par la CJUE avec le contrôle externe la CEDH (1.) mais aussi celle de la mutation nécessaire du contrôle interne (2.).

\section{L'articulation des contrôles juridictionnels internes et externes}

La question est devenue classique : le fonctionnement de l'espace pénal européen n'appelle-t-il pas aujourd'hui un contrôle direct de la part de la Cour européenne des droits de l'Homme, au vu de son impact sur les droits fondamentaux des personnes? Après avoir été posée en termes de concurrence juridictionnelle, sinon de rapports de force, l'interrogation porte aujourd'hui sur la complémentarité des solutions qu'apportera l'adhésion de 1 'Union à la CEDH.

\section{- La concurrence du contrôle externe}

L'intervention de la Cour européenne des droits de l'Homme dans le contentieux de l'espace pénal européen n'a jamais été une simple éventualité, quand bien même l'Union ne serait pas partie à la CEDH. Il n'y a pas grand étonnement à le répéter dans la mesure où la Cour européenne contrôle l'action individuelle des Etats parties en matière répressive à divers égards.

Assujettir l'Union à un contrôle du même type n'est plus un objet de débat aujourd'hui, depuis la fameuse jurisprudence Bosphorus. ${ }^{45}$ Par principe, les mesures nationales d'exécution des politiques d'entraide répressive de l'Union relèvent bien du contrôle du juge de Strasbourg dans la mesure où les Etats membres qui les appliquent sont également liés par le respect de la CEDH. Les précisions apportées par la Cour européenne des droits de l'Homme ont simplement dessiné les lignes rouges à ne pas franchir sous peine de voir la Cour opérer un contrôle indirect mais bien réel de la conformité de la politique sécuritaire de l'Union aux droits fondamentaux. La Cour européenne l'a exprimé en indiquant que les Etats membres «sont responsables au titre de l'article 1 de la Convention de tous les actes et omissions de leurs organes, qu'ils découlent du droit interne ou de la nécessité d'observer des obligations juridiques internationales. Ledit texte ne fait aucune distinction quant au type de normes ou de mesures en cause et ne soustrait aucune partie de la "juridiction » des Parties contractantes à l'empire de la Convention ». ${ }^{46}$ Aussi, « de l'avis de la Cour, une mesure de l'Etat prise en exécution de pareilles obligations juridiques doit être réputée justifiée dès lors qu'il est constant que l'organisation en question accorde aux droits fondamentaux (cette notion recouvrant à la fois les garanties substantielles offertes et les mécanismes censés en contrôler le respect) une protection à tout le moins équivalente à celle assurée par la Convention $\gg{ }^{47}$ Cette présomption peut néanmoins être renversée et justifier l'intervention de la

45 CEDH, arrêt du 30 juin 2005, Bosphorus c. Irlande.

$46 \S 153$.

$47 \S 155$. 
Cour européenne « si l'on estime que la protection des droits garantis par la Convention était entachée d'une insuffisance manifeste $»{ }^{48}$

Or, les conditions dans lesquelles le juge de l'Union était amené à intervenir dans le cadre de l'espace pénal européen nourrissaient largement les doutes et l'opinion concordante mais dissonante exprimée par certains juges dans l'affaire Bosphorus accréditait les soupçons, au vu des restrictions de l'accès des particuliers au juge de l'Union. Du reste, le juge communautaire d'avant l'arrêt Kadi l'avouait sans être démenti très vigoureusement lors d'un pourvoi devant la $\mathrm{CJCE}^{49}$ : des requérants individuels inscrits sur des listes antiterroristes «ne disposent probablement d'aucun recours juridictionnel effectif $\gg^{50}$ dans l'Union. L'un des avocats généraux n'hésitait d'ailleurs pas à qualifier ce défaut de protection juridictionnelle " d'incohérence systémique très grave et flagrante sur le plan interne à l'Union $\gg .{ }^{51}$ Les efforts de la CJCE pour desserrer l'étreinte dans sa jurisprudence ont, heureusement, été relayés par le traité de Lisbonne, comme on l'a vu plus haut.

Il était temps. Une seconde jurisprudence de la CEDH tout aussi déstabilisante que la jurisprudence Bosphorus est en effet intervenue récemment pour accroître la pression. Dans son arrêt $M S S$ c. Belgique et Grèce ${ }^{52}$ concernant le transfert de demandeurs d'asile entre Etats membres de l'Union sur la base de la réglementation « Dublin », la Cour européenne des droits de l'Homme clarifie les enjeux, si besoin en était encore. A l'occasion de l'application d'un règlement communautaire et parce qu'un autre Etat partie l'y invitait, la Cour précise le sens qu'il convient de donner à sa jurisprudence Bosphorus en cas de mesure d'éloignement entre Etats membres sur la base d'un texte de droit de l'Union. Elle rappelle que si la Convention n'interdit pas aux Parties contractantes de transférer des pouvoirs souverains à une organisation internationale à des fins de coopération dans certains domaines d'activité, "les Etats demeurent néanmoins responsables au regard de la Convention de tous les actes et omissions de leurs organes qui découlent du droit interne ou de la nécessité d'observer les obligations juridiques internationales. Une mesure de l'Etat prise en exécution de pareilles obligations juridiques doit être réputée justifiée dès lors qu'il est constant que l'organisation en question accorde aux droits fondamentaux une protection à tout, le moins équivalente à celle assurée par la Convention ». Cette présomption ne le délie pas de ses obligations «notamment lorsqu'il a exercé un pouvoir d'appréciation ». Elle prend d'ailleurs soin d'ajouter que la présomption d'équivalence ne valait pas pour l'ensemble du droit de l'Union : "pour parvenir à cette conclusion, elle a attaché une grande importance au rôle et aux compétences de la CJCE, devenue CJUE, en la matière, considérant en effet que l'effectivité des garanties matérielles des droits fondamentaux dépendait des mécanismes de contrôle mis en place pour assurer leur respect. La Cour a pris soin aussi de limiter la portée de l'arrêt Bosphorus au droit communautaire au sens strict, à l'époque le " premier pilier» du droit de l'Union européenne ». ${ }^{53}$ Condamnant la

$48 \S 156$.

49 CJCE, 27 février 2007, Segi et al., C-355/04 P, point 51.

50 TPI, 7 juin 2004, Segi et al., T-338/02, point 18.

51 Conclusions Mengozzi sous CJCE, Segi et al., C-355/04 P, 26 octobre 2006, point 85.

52 CEDH, 21 janvier 2011, M.S.S. c. Belgique et Grèce, req. 30696/09.

53 point 338 . 
Belgique, elle invite donc les Etats à la plus grande vigilance mutuelle et à s'interroger en cas de besoin sur la situation dans l'Etat de membre de destination...

En transposant le raisonnement à l'espace pénal européen, on mesure la fragilité du droit de l'Union et les conséquences qui en résultent pour les Etats membres lorsqu'ils prétendent automatiser leurs réactions au nom de la confiance mutuelle qu'ils se prêtent. La Cour rappelle du reste « que lorsque des Etats coopèrent dans un domaine où la protection des droits fondamentaux peut se trouver affectée, il est contraire au but et à l'objet de la Convention qu'ils soient exonérés de toute responsabilité au regard de la Convention dans le domaine d'activité concerné (voir parmi d'autres: Waite et Kennedy c. Allemagne [GC], no 26083/94, § 67, CEDH 1999-I) ». ${ }^{54}$ Il n'y a donc aucune raison de voir le juge européen baisser ici la garde au vu de sa jurisprudence habituelle en matière d'extradition.

Sans doute n'est-ce pas un hasard si la Cour européenne prend la peine à cette occasion de $\operatorname{citer}^{55}$ sa propre décision dans l'affaire Stapleton c. Irlande, ${ }^{56}$ qui semble confirmer l'applicabilité de cette ligne jurisprudentielle au droit de l'espace pénal européen. Dans cette affaire concernant un mandat d'arrêt européen visant un ressortissant irlandais émis par le Royaume Uni, le requérant mettait en cause le respect de l'article 6 de la CEDH et les règles du procès équitable, principalement pour des raisons de délais. Le juge irlandais lui avait donné raison avant d'être contredit par la Cour suprême irlandaise en s'appuyant tout à la fois sur la décision cadre telle qu'interprétée par la CJUE dans l'affaire Advocaten, sur le principe de confiance mutuelle de l'arrêt Pupino, et sur le fait que des recours existaient en droit britannique.

La Cour européenne approuve cette position avec un sens des nuances qu'il importe de noter. Elle « rappelle que, le droit à un procès équitable en matière pénale consacré par l'article 6 occupant une place éminente dans les sociétés démocratiques, elle n'exclut pas qu'une décision d'extradition puisse exceptionnellement soulever un problème sur le terrain de ce texte au cas où le fugitif aurait subi ou risquerait de subir un déni de justice flagrant dans l'Etat requérant (Soering c. Royaume-Uni, 7 juillet 1989, § 113, série A no 161, et Mamatkoulov et Askarov c. Turquie [GC], nos 46827/99 et 46951/99, $\S 88, C E D H 2005-I) »$.

Cette réserve n'est pas nouvelle, loin de là en matière extraditionnelle. La Cour manifeste d'ordinaire sa distance avec le droit de l'extradition qui, par lui-même, n'est pas garanti par la Convention et lui a donné l'occasion de développer sa théorie de la « protection par ricochet ». Elle en a fait application de manière explicite à propos du mandat d'arrêt européen, en deux temps dans une décision apparemment très claire. ${ }^{57}$ En premier lieu, la Cour y rappelait d'emblée que le droit de ne pas être extradé ne figure pas, comme tel, au nombre des droits et libertés reconnus dans la Convention et ses Protocoles additionnels. Par ailleurs, la procédure d'extradition n'a pas trait à une contestation sur les droits et obligations de caractère civil du requérant, ni au bien-fondé d'une accusation en matière pénale dirigée contre lui au sens de l'article 6 de la Convention. ${ }^{58}$ Elle avait ensuite transposé le raisonnement au mandat d'arrêt européen : « la

54 point 342 .

55 point 343 .

56 CEDH, décision du 4 mai 2010, Stapleton c. Irlande, req. 56588/07.

57 CEDH, décision du 7 octobre 2008, Monedero Angora c. Espagne, req. 41138/05.

58 CEDH, décision du 16 avril 2002, Peñafiel Salgado c. Espagne, req. 65964/01. 
Cour constate que la procédure du mandat d'arrêt européen remplace la procédure classique d'extradition entre les Etats membres de l'Union européenne et poursuit le même but, à savoir la remise aux autorités de l'Etat requérant d'une personne qui est soupçonnée d'avoir commis une infraction ou qui tente d'échapper à l'action de la justice après avoir été condamnée par une décision définitive. L'exécution du mandat d'arrêt européen est par ailleurs pratiquement automatique : l'autorité judiciaire ne procède pas à un nouvel examen du mandat pour vérifier sa conformité avec sa propre législation interne, et n'en refuse l'exécution que pour des motifs fixés par la loi. Il découle de ce qui précède, analysé à la lumière de la jurisprudence de la Cour, que cette procédure ne porte pas sur le bien-fondé d'une accusation en matière pénale. Il s'ensuit que cette partie de la requête est incompatible rationae materiae avec les dispositions de la Convention au sens de l'article $35 \S 3$, et doit être rejetée en application de l'article $35 \S 4$ ».

Tel n'est pas tout à fait l'enseignement de la jurisprudence Stapleton précitée puisque la Cour y entrouvre la voie à un contrôle de l'Etat d'émission. Rejetant l'argument selon lequel « lequel l'Etat exécutant ou transférant ne devrait pas se borner à rechercher un " déni flagrant » mais déterminer s'il a été établi qu'existait dans l'Etat d'émission (en l'espèce, le Royaume-Uni) un risque réel d'inéquité de la procédure pénale », elle n'écarte donc pas par principe l'idée de cette recherche. Elle « souscrit » d'ailleurs au raisonnement de la Cour suprême irlandaise selon lequel les juridictions britanniques sont les mieux placées pour apprécier l'équité de la procédure. Enfin, elle écarte avec une brutalité qui ne serait plus de mise après l'arrêt $M S S$ « la thèse du requérant selon laquelle il est fondé à faire protéger ses droits garantis par la Convention dès que la question se pose (en l'espèce, devant les juridictions irlandaises) est également déplacée, eu égard au fait que le Royaume-Uni est partie à la Convention ». ${ }^{59}$

Sans franchir le pas, principalement en raison de la nature des griefs en cause, ${ }^{60}$ le juge de Strasbourg n'avalise pas pour autant l'automaticité de la reconnaissance mutuelle.

C'est dire l'urgence d'une meilleure articulation des dispositifs européens de protection, que l'adhésion prochaine de l'Union à la CEDH devrait réaliser.

\section{- La complémentarité des contrôles}

A la veille de l'adhésion programmée de l'Union européenne à la CEDH, la question n'est pas tant de raisonner en termes de préséance ou de supériorité d'un texte ou d'une juridiction sur l'autre que d'essayer d'en évaluer les conséquences pour l'espace pénal européen.

Les lignes générales du dispositif envisagé semblent être suffisamment connues pour appeler un bref commentaire. Le schéma envisagé comporte en effet deux novations susceptibles de garantir une complémentarité satisfaisant les intérêts de l'espace pénal européen, parce que prenant en compte le jeu des compétences en présence. En effet, soit la violation de la Convention est le fait d'un Etat membre isolément, et il doit en répondre directement comme c'est actuellement le cas, soit cette violation est le fait d'un acte de l'Union ou de ses organes et elle doit en répondre désormais, une fois que les voies de recours du contentieux de l'Union ont été épuisées.

59 point 30

60 L'article 6 et non pas les articles 2 ou 3 de la CEDH. 
La première nouveauté concerne le rôle dévolu à la Cour de justice dans le processus de contrôle. En préalable à l'intervention de la Cour européenne des droits de l'Homme et en écho au souci exprimé ouvertement par la CJUE de voir préserver le jeu des renvois préjudiciels qui permettent normalement de purger le droit de l'Union de toute illégalité, un mécanisme interne d'intervention de la CJUE préalable à la saisine de la CEDH est envisagé. Reprenant la suggestion du juge Timmermans devant la commission des affaires constitutionnelles du Parlement européen, l'idée d'un mécanisme garantissant que la CJUE puisse contrôler la conformité du droit de l'Union aux droits fondamentaux est acquise, avec l'appui non négligeable des présidents des deux Cours.

La CJUE ne serait pas automatiquement saisie mais sur demande du requérant ou de la Commission selon des schémas à déterminer et une procédure accélérée. Sa décision n'aurait pas d'effet direct mais indirect sur la procédure devant la CEDH, celle-ci étant néanmoins évidemment conduite à en observer les conséquences. Ainsi, en cas d'invalidation de l'acte de l'Union incriminé et en fonction des conséquences attachées par le droit national à cette invalidation, le requérant pourrait voir sa situation rétablie et perdre son statut de victime au sens de la Convention.

Garantir de manière contentieuse la conventionalité du droit de l'Union européenne implique également que l'Union puisse présenter sa défense devant l'organe de contrôle. L'institution d'un mécanisme de co-défendeur constitue donc la seconde innovation du processus en cours. Elle aurait pour principal avantage, en plaçant l'Union aux cotés de ses Etats membres, d'éviter de placer la Cour européenne en arbitre des conflits de compétence qui jalonnent la vie des traités constitutifs de l'Union. En effet, la particularité du système de l'Union européenne veut que le droit de l'Union, qui prime sur le droit interne, soit mis en œuvre par les Etats membres ou, plus rarement, par ses propres organes. L'espace pénal européen illustre cette spécificité à merveille puisque les règles gouvernant l'entraide répressive sont tour à tour susceptibles d'application par les Etats membres de l'Union comme par ses agences que sont Europol ou Eurojust. Dès lors, la participation de l'Union aux procédures devant la CEDH aux cotés des Etats membres permettrait d'établir en toute connaissance de cause la conformité de l'action de l'Union à la $\mathrm{CEDH}$ et de renvoyer ensuite à l'Union et à ses membres le soin d'en tirer les conséquences.

Cependant et quoi qu'il en soit, il n'est pas certain que cette meilleure complémentarité des contrôles soit aussi révolutionnaire que certains le prétendent. A n'en pas douter, le principal bénéfice à en attendre est vraisemblablement celui de la cohérence du niveau de protection attendu, davantage que celui du fond. C'est dire qu'il faut peut-être nourrir davantage d'espoir dans une mutation nécessaire du dispositif existant.

\section{La mutation nécessaire du contrôle juridictionnel interne de l'espace pénal}

Malgré l'influence incontestable que la CEDH peut jouer dans le respect des droits fondamentaux, la situation est loin d'être satisfaisante dans l'UE à ce jour. Loin de chercher à « judiciariser » par principe ou effet de mode, il convient de réfléchir alors aux besoins de l'espace pénal européen. Ceux-ci s'expriment aussi bien à partir de facteurs extérieurs, guidant une évolution logique, que de facteurs propres à l'espace répressif commun et à son incapacité à remplir ses objectifs. 


\section{- Une mutation logique}

Le diagnostic est difficile à contester. La disparité du niveau de la protection des droits fondamentaux dans l'Union fait courir un risque sérieux à la cohésion de l'espace pénal européen. Cette disparité peut être une source de blocage sérieux de cet espace ou, au mieux de ralentissement sérieux. Les sources d'interrogations en ce sens sont nombreuses et la solution textuelle représentée par l'adoption de la Charte des droits fondamentaux pas davantage que l'adhésion à la CEDH n'offrent les réponses miraculeuses que l'on peut croire. Accroitre la pression judiciaire peut s'avérer être de meilleur profit, pour peu que l'on s'accorde sur le sens à donner à ce degré supplémentaire de « judiciarisation ». Dans la logique des constats posés précédemment, c'est au delà de la fonction « constitutionnelle » du juge qu'il faut sans doute aller chercher.

Tirer les conséquences des difficultés présentes est une première démarche. La pression de l'adhésion ne doit pas être mésestimée de ce point de vue, y compris au plan matériel. Ainsi, les immunités contentieuses âprement disputées par les Etats membres depuis Maastricht méritent-elles un regard nouveau. De la réserve de compétence en matière d'ordre public et de sécurité publique à la préservation de la $\mathrm{PESC}$, que resterat-il après confrontation avec le droit de la CEDH? A l'opposé, la cour constitutionnelle qu'est la CJUE ne sera-t-elle pas conduite à importer un peu de subjectivisme dans ses raisonnements, à conduire ceux-ci au regard « des » droits et pas seulement « du droit»? La « marge nationale d'appréciation » étatique qui n'est pas vraiment dans sa culture, à l'inverse du droit de la CEDH, et qui a la préférence des Etats si l'on en croit leurs explications du blocage du texte sur les garanties procédurales des suspects, trouverat-elle droit de cité dans le droit de l'Union?

Il convient donc d'assumer les implications du mouvement qui s'enclenche. Concrètement, en effet, c'est aujourd'hui le juge et non les textes qui posent question au développement de l'espace pénal européen. Lorsque, durant les longs mois de l'affaire Ramda, le juge britannique estime insuffisantes les garanties du système judiciaire français pour procéder à la livraison du financier des attentats du métro Saint Michel à Paris avant de changer d'attitude quelques mois plus tard, à droit constant mais après les attentats du métro londonien, ce n'est pas le droit qui pose problème, c'est la lecture qu'en fait le juge national. Lorsque les plus hautes autorités du même Etat membre, présidant alors l'Union européenne, mettent expressément en cause la jurisprudence protectrice de la CEDH sur la détention des terroristes et l'interdiction de la torture et des traitements dégradants face aux impératifs de la lutte anti-terroriste, c'est toujours le juge qui est dans l'oeil du cyclone et refuse, heureusement, de déférer à l'injonction. ${ }^{61}$

Voir le juge de l'Union camper sur ses fonctions traditionnelles, sans que cela soit pour autant un signe de prudence ou de pusillanimité, fragilise sans doute une part de la construction en cours. D'où le besoin d'une mutation ou d'une accentuation de la part « judiciaire » de l'espace pénal européen si l'Union souhaite éviter l'écueil de la renationalisation de cet espace par le juge interne. La porte est en effet étroite entre l'absence de contrôle, peu envisageable et dangereuse pour les droits fondamentaux, et

61 CEDH, 28 février 2008, Saadi c. Italie. 
la généralisation de ce dernier, risquée pour le fonctionnement de l'espace commun. L'observation des systèmes pénaux nationaux démontre amplement, si besoin en était, la variabilité de la lecture des garanties fondamentales à l'intérieur d'un même système pénal. Comment n'en irait-il pas de la même façon et pire encore à l'échelle de l'Union? On devine aisément ce qu'il en résulterait si le juge national était investi d'un droit de regard sur le voisin... Le risque de voir un Etat membre utiliser par exemple le nombre des condamnations d'un Etat partie à la CEDH sur le terrain de l'article 6 pour refuser de déférer aux impératifs de la coopération répressive dans l'espace pénal européen n'est pas illusoire, et il faut l'anticiper. Il faut donc évoluer par le haut, c'est-à-dire en se penchant sur les fonctions de la juridiction de l'Union européenne.

La question n'est pas de s'interroger sur la capacité de tel ou tel système de protéger au mieux les droits fondamentaux, comme on fait souvent le choix pour aborder la difficulté. Il faut, au contraire réfléchir aux évolutions indispensables à l'efficacité de l'espace pénal commun. Autrement dit, outre la consolidation du contrôle juridictionnel entamée en parallèle par le juge de l'Union et les auteurs du traité de Lisbonne, l'Union européenne est-elle capable de relever le défi de la spécialisation d'un contrôle adapté à la matière c'est-à-dire à cet espace pénal profondément original?

Distinguer le possible du souhaitable n'empêche pas de pointer des insuffisances majeures du dispositif actuel, même s'il est en voie d'adaptation avec l'adhésion à la CEDH. Si l'espace pénal européen dispose bien d'instances judiciaires au plein sens du terme à Luxembourg comme à Strasbourg et malgré les fausses querelles sur leur composition, elles n'apportent pas les réponses satisfaisantes.

L'épée de Damoclès tenue par le juge de la CEDH a été et demeure d'un apport sans pareil, chacun en a conscience, tout comme il faut en avoir les limites à l'esprit. La première est structurelle, malgré les efforts de rénovation auxquels les Etats parties ont consenti depuis plus d'une décennie. La lenteur de la procédure de contrôle, la portée déclaratoire des jugements de la Cour renvoyant aux Etats parties le soin de remédier aux violations constatées ne mettent pas la $\mathrm{CEDH}$ en situation de répondre pleinement aux besoins constatés et de satisfaire l'urgence de la lutte contre le crime. La seconde limite est fonctionnelle et elle avait, pour une fois, été correctement exprimée par certains Etats membres lors de leur tentative réussie de blocage de la réglementation des droits procéduraux. Préférer le cadre de la CEDH à celui de l'Union s'expliquait selon eux par la volonté de conserver la marge d'appréciation dont ils disposent dans le premier des deux cadres... Refuser de les suivre sur ce terrain conduit alors à aller de l'avant.

La progression des conditions de l'intervention de la CJUE dans le cadre de l'espace pénal européen ne lève pas toutes les interrogations, loin de là. Outre la mise à niveau de la protection juridictionnelle dans l'Union opérée par le traité de Lisbonne, l'instauration d'une procédure préjudicielle d'urgence (PPU) ${ }^{62}$ en témoigne. Typiquement instituée pour pallier les lenteurs des procédures de droit commun, ${ }^{63}$ elle s'ajoute à l'injonction du nouvel article $267 \S 3$ du TFUE relatif à la procédure préjudicielle qui dispose que « si une telle question est soulevée dans une affaire pendante devant une juridiction

62 Décision du Conseil du 20 décembre 2007 portant modification du Protocole sur le statut de la Cour de justice, JO L 24 du 29 janvier 2008 p. 39.

63 Deux mois et demi pour en connaître contre dix huit mois pour une question préjudicielle classique. Sur le thème, voir A. Tizzano et B. Gencarelli, « La procédure préjudicielle d'urgence devant la CJUE », Mélanges en l'honneur de JP Jacqué, Dalloz 2010 p. 639. 
nationale concernant une personne détenue, la Cour statue dans les plus brefs délais ». On serait alors en droit d'imaginer que l'invitation au dialogue adressée au juge national soit suivie d'effets.

Tel n'est pas le cas et sans doute faut-il y remédier pour un fonctionnement harmonieux de l'espace pénal européen. Constater que seulement 38 renvois préjudiciels introduits sur 381 en 2010 et que 24 affaires préjudicielles clôturées sur 482 concernent l'ELSJ pose une question sérieuse quant au maniement du droit de l'Union par le juge national dans ce domaine. Comptabiliser seulement une PPU en 2009 et aucune en 2010 interroge tout autant...D'autant que, en affinant la réflexion à propos des PPU, ${ }^{64}$ un constat un peu inattendu indique que, somme toute, le juge civil trouve à cette procédure un intérêt à la mesure de l'indifférence du juge pénal. Carence ou échec, le dialogue entre le juge pénal national et le juge de l'Union n'est pas ce qu'il devrait être, loin de là et au vu des difficultés potentiellement décelables en ce domaine. Rapporté aux 15827 mandats d'arrêt européen émis dans l'Union et aux 4431 MAE exécutés en 2009, l'autisme du juge pénal national est trop marqué pour que l'on puisse le passer sous silence. Il faut en prendre conscience dès à présent : le jeu de la reconnaissance mutuelle et de l'équivalence qui la sous-tend réclame un contrôle de « qualité » qui fait défaut aujourd'hui.

Dès lors, si l'appui sur le juge national n'est pas ce qu'il devrait logiquement être, il convient de réfléchir à une accentuation du rôle du juge de l’Union.

\section{- Un contrôle autonome}

Banaliser ou spécialiser la judiciarisation de l'espace pénal soulève des questions de fond. Le principe de réalité ne suffit pas à les écarter, au prétexte que l'état politique de l'Union européenne rendrait la réflexion purement théorique. Au regard des dysfonctionnements actuels et de la menace qu'ils contribuent à faire peser sur le jeu harmonieux de l'espace pénal européen, la réflexion mérite d'être ouverte.

Il ne s'agit évidemment pas de s'interroger sur les fonctions classiques du juge de l'Union européenne. Elles font peu ou prou consensus et elles ne sont remises en question par personne. En revanche, demeure ouverte la question de l'exacte dimension judiciaire de l'espace pénal européen, spécialisée ou non, surtout au vu de la collaboration préjudicielle en matière pénale ou de son succès très relatif, comme on voudra. L'existant comme les évolutions potentielles de l'espace pénal commun appellent un approfondissement de cette « judiciarisation » et creusent la voie d'une intervention plus poussée de la Cour de justice. En d'autres termes, ce mouvement irréversible ajouté au pluralisme juridique caractérisant désormais l'Union nécessite de sortir des approches horizontales ou hiérarchiques dont le confort satisfaisait l'observateur jusqu'ici. Un autre type de relations doit s'instaurer entre le juge national et le juge de l'Union.

Les objets de réflexion sont connu : un principe de fonctionnement, la reconnaissance mutuelle, et un début d'action opérationnelle de l'Union où des acteurs d'une grande originalité montent en puissance, d'Europol à Eurojust.

64 En matière civile, à ce jour, on peut recenser 7 affaires (Rinau C-195/08 PPU; Kadzoev C-357/09 PPU; Detiček C-403/09 PPU; Povse C-211/10 PPU; McB C-400/10 PPU; Mercredi C-497/10 PPU; Aguirre Zarraga C-490/10 PPU) pour une en matière migratoire (El Dridi C-61/11 PPU) et seulement 2 en matière pénale (Goicoechea C-296/08 PPU; Leyman C-388/08 PPU). 
- Le jeu de la reconnaissance mutuelle, en premier lieu, appelle un regard spécifique du juge, au regard de la pression exercée par les droits fondamentaux dans sa gestion. Qu'il s'agisse du principe ne bis in idem ou du contrôle de la proportionnalité de la demande d'entraide, ${ }^{65}$ se satisfaire des déséquilibres existants semble difficile dans la durée. La pétition de principe initiale consistant à affirmer comme étant acquis le respect mutuel des droits fondamentaux paraît avoir été affaiblie tant par la pratique que par la vigilance due au respect de la jurisprudence précitée $M S S$. Les institutions de l'Union européenne en ont d'ailleurs parfaitement conscience et la Commission en convient dans son évaluation récente du mandat d'arrêt européen. ${ }^{66}$

Benoîtement, le Conseil avalise l'importance de ces doutes puisque, dans le préambule de la directive 2010/64 relative à l'interprétation et à la traduction dans le cadre des procédures pénales, il constate que «bien que tous les États membres soient parties à la CEDH, l'expérience a montré que cette adhésion, à elle seule, ne permet pas toujours d'assurer un degré de confiance suffisant dans les systèmes de justice pénale des autres États membres $\gg .{ }^{67} \mathrm{~A}$ n'en pas douter aujourd'hui, l'hypothèse d'une jurisprudence $N . S^{68}$ où le juge de l'Union constate la présence d'une « présomption réfragable de respect des droits fondamentaux » par un Etat membre en matière d'asile n'est pas inimaginable en matière pénale.

Pourtant, si la mise en œuvre d'un espace unique dans lequel l'action répressive s'automatise peut s'accommoder de dysfonctionnements du point de vue technique, ${ }^{69}$ elle ne peut en faire autant sur le plan politique.

Du point de vue des justiciables, l'Union ne peut passer outre les implications de l'unité répressive de l'espace commun qu'elle s'efforce d'établir. La confiance mutuelle fondant cet espace et qui permet son fonctionnement réclame un contrôle judiciaire car l'unité répressive recherchée doit être accompagnée de garanties individuelles. Elle est en effet susceptible de menacer les droits individuels car chaque justiciable a vocation a bénéficier d'un traitement équitable et qu'un obstacle soit dressé face à l'arbitraire. Qu'il s'agisse de ne pas être jugé deux fois pour les mêmes faits ou de pouvoir conserver l'équivalent des garanties fondamentales fournies par son propre système national, chaque individu devrait être en droit de disposer d'un interlocuteur judiciaire ${ }^{70}$ pour faire valoir sa position.

Le jeu des droits fondamentaux dans l'espace pénal commun accentue donc la pression en faveur de la « judiciarisation ». Il appelle un renforcement effectif de la garantie judiciaire, au nom de la séparation des pouvoirs et nécessite que la personne concernée puisse accéder à un juge autre que le juge national, qui le réclame ou qui le livre. Il va

65 Qui semble aujourd'hui s'insérer comme un correctif du principe, à en croire la Commission dans son dernier rapport d'évaluation sur le mandat d'arrêt européen.

66 COM (2011) 175 précité.

67 Voir le considérant 6 de la directive 2010/64 du 20 octobre 2010 relative au droit à l'interprétation et à la traduction dans le cadre des procédures pénales, JO L 280 du 26 octobre 2010 p. 1.

68 CJUE, 21 décembre 2011, N.S., C- 411/10.

69 Au prix de sa seule efficacité.

70 D. Flore note justement dans une étude remarquable que le contrôle d'Eurojust sur l'exercice des compétences s'apparente davantage à un arbitrage, « Des organes juridictionnels en matière pénale pour l'Union européenne » CDE 2003 p. 614. 
de soi qu'un tel contrôle ne pourrait porter que sur la légalité et non sur les éléments factuels mais il ne doit pas être écarté dans son principe. On peut ainsi, à terme, imaginer qu'il soit institué par exemple, comme cela avait été évoqué, ${ }^{71}$ au moyen d'une chambre pénale instituée au sein de la CJUE. Qu'il s'agisse de régler le jeu du droit fondamental à ne pas être jugé deux fois ou alors de trancher une divergence sur l'état des droits fondamentaux dans l'Etat d'émission d'une demande d'entraide, seul le juge de l'Union est susceptible d'apporter une réponse crédible et incontestable. Tout comme il est le seul à déterminer le seuil d'intensité ou de proportionnalité à partir duquel des techniques telles que le mandat d'arrêt européen ont vocation à jouer, sachant que les questions soulevées ne sont pas du tout de même nature selon que l'hypothèse d'une enquête pénale ou de l'exécution d'un jugement est en jeu. ${ }^{72}$

La Commission n'avait d'ailleurs pas écarté l'éventualité d'un refus sur ce point dès son premier rapport sur le mandat d'arrêt européen, tout en lui conservant un caractère « exceptionnel $» .{ }^{73}$ On imagine difficilement que le juge national puisse légitimement effectuer ce contrôle sans recours au juge de l'Union, sous peine de disparités fortement dommageables pour l'espace pénal commun. On imagine également que la relation entre le juge de l'Union et ce justiciable soit encore améliorée, tant il est vrai, en particulier dans le contentieux préjudiciel, que l'absence de lien direct entre les parties et le juge pose ici problème dans le monde pénal. Car, et c'est un élément non négligeable du débat avec lequel il faut composer, la légitimité de l'intégration européenne et de son juge demeure encore à affermir sinon à établir. ${ }^{74}$

Encore faut-il s'accorder sur l'identification et la portée de ces droits et que le législateur de l'Union incite à cette garantie. L'adoption par le Conseil d'une feuille de route visant à renforcer les droits procéduraux des suspects liste les questions sensibles ${ }^{75}$ parce que susceptibles de poser problème au fonctionnement harmonieux de l'espace pénal commun. Elle témoigne en réalité de la carence actuelle du système et de son incapacité à apporter rapidement des réponses aux difficultés rencontrées.

71 Voir le Livre vert de la Commission sur les conflits de compétence et le principe $\mathrm{Ne}$ bis in idem dans le cadre des procédures pénales, COM (2005) 696.

72 S. de Biolley, «Quelle contribution de la Cour de justice à l'espace pénal européen demain » in Le contrôle juridictionnel dans l'espace pénal européen, S. Braum et A. Weyembergh (dir.), ed. de l'ULB, 2009 p. 320.

73 COM 2005 (63) du 23 février 2005 p. 6 : « le Conseil n'a pas entendu faire de la condition générale de respect des droits fondamentaux un motif explicite de refus en cas de violation. Cependant il va sans dire qu'une autorité judiciaire est toujours fondée à refuser l'exécution d'un mandat d'arrêt si elle constate que la procédure est entachée d'une violation de l'article 6 TUE et des principes constitutionnels communs aux Etats membres; dans un système fondé sur la confiance mutuelle, une telle situation devrait rester exceptionnelle $»$.

74 Pour des interrogations critiques : PA Albrecht, « Le pouvoir judiciaire indépendant comme contrepoids à l'érosion des principes européens de droit pénal » in Le contrôle juridictionnel dans l'espace pénal européen, S. Braum et A. Weyembergh (dir.), ed. de l'ULB, 2009 p. 145; voir également les conclusions de S. Braum, p. 331.

75 Outre la directive précitée, il faut y ajouter le droit d'être informé de ses droits (déclaration des droits); le droit à l'assistance d'un conseiller juridique avant et pendant le procès; le droit pour une personne détenue de communiquer avec les membres de sa famille, ses employeurs et les autorités consulaires; la protection des suspects vulnérables. Un livre vert sur la détention provisoire est également évoqué. 
- La dimension opérationnelle de l'entraide répressive dans l'Union accentue ce besoin d'une réponse juridictionnelle appropriée, à la fois encore du point de vue de l'individu et de la sécurité des procédures. Le jeu des équipes communes d'enquête qui sont susceptibles de fournir des éléments d'information, la montée en puissance prévisible d'Europol, la mise en œuvre des prérogatives d'Eurojust sans parler des évolutions à venir telles que l'évolution d'Eurojust vers un ministère public européen contribue à nourrir l'interrogation. Autrement dit, toute dépossession des souverainetés pénales nationales doit s'accompagner d'un contrôle judiciaire correspondant à la marge d'autonomie des acteurs de l'espace pénal qui sont investis : la " résolution » de conflits de compétences, le contrôle des décisions d'un futur Parquet européen, la « réalisation » d'enquêtes prévues par le traité de Lisbonne sont autant d'exemple d'une judiciarisation souhaitable.

En conclusion, la « judiciarisation » de l'espace pénal européen apparaît comme inévitable. Rencontrant logiquement les obstacles habituels à toute progression de l'intégration européenne, cette montée en puissance de l'institution judiciaire n'en pose pas moins une question fondamentale, celle de la priorité donnée jusqu'ici au traitement juridictionnel des questions institutionnelles.

La banalisation de l'entraide répressive au sein de l'Union européenne réclame un accès élargi du justiciable au juge. Elle ne peut donc que s'accompagner d'une montée en puissance de l'institution judiciaire, dont on conçoit difficilement qu'elle puisse n'être que nationale. Sous-jacente depuis les origines de l'espace pénal européen, la question relève du tabou ou du non-dit. Il n'est pas certain que cette posture de l'autruche puisse être tenue durablement, à l'instant où les premières critiques frappant cet espace pénal et ses instruments commencent à se faire jour ${ }^{76} \ldots$

76 La campagne menée à l'encontre du mandat d'arrêt européen dans le cadre de la lutte contre le terrorisme au Pays basque est révélatrice des limites de l'exercice. Hommes politiques ayant parfois voté la révision constitutionnelle de 2003, militants nationalistes ou simples citoyens y font le procès d'un procédé « politique » portant atteinte à des principes essentiels tels que la non-livraison des nationaux alors que, précisément, le mandat d'arrêt européen a été institué pour l'inverse....Méconnaissance de l'appui du juge de l'Union par le juge d'exécution et activisme du juge d'émission expliquent largement ce rejet paradoxal mais significatif. 